\title{
La rappresentazione cartografica del paesaggio fra arte e geometria
}

\section{Luisa Rossi}

\section{(2) OpenEdition \\ 1 Journals}

Edizione digitale

URL: http://journals.openedition.org/edl/515

DOI: $10.4000 /$ edl. 515

ISSN: 2296-5084

\section{Editore}

Université de Lausanne

\section{Edizione cartacea}

Data di pubblicazione: 15 mai 2013

Paginazione: 305-322

ISBN: 978-2-940331-32-1

ISSN: 0014-2026

Notizia bibliografica digitale

Luisa Rossi, «La rappresentazione cartografica del paesaggio fra arte e geometria », Études de lettres [Online], 1-2 | 2013, online dal 15 mai 2016, consultato il 18 décembre 2020. URL : http:// journals.openedition.org/edl/515; DOI : https://doi.org/10.4000/edl.515 


\section{LA RAPPRESENTAZIONE CARTOGRAFICA DEL PAESAGGIO FRA ARTE E GEOMETRIA ${ }^{1}$}

Entre le XVII e et le XIX ${ }^{\mathrm{e}}$ siècle, la cartographie occidentale inclut la représentation du paysage et en particulier du relief terrestre. Dans les cartes géographiques du XVII ${ }^{\mathrm{e}}$ et du XVIII e siècle, la perspective cavalière domine par son adoption "schizophrénique» de deux points de vue: vertical pour les éléments planimétriques et horizontal pour les éléments altimétriques. Le paysage se trouve ainsi au croisement entre représentation artistique et technique, entre imitation naturaliste et abstraction géométrique. Au début du XIX $\mathrm{X}^{\mathrm{e}}$ siècle, suite à l'invention des courbes de niveau, les milieux scientifiques et militaires de la France napoléonienne s'orientent vers l'adoption d'une cartographie exclusivement abstraite. L'article étudie ce moment crucial à partir des documents liés à la réalisation du plan-relief du Golfe de la Spezia, confiée par l'empereur à Pierre-Antoine Clerc, capitaine du Génie, et à sa brigade topographique. Il s'agit d'un vaste corpus qui montre une fusion parfaitement équilibrée entre figuration picturale et rigueur géométrique.

In un volume collettivo dedicato pochi anni fa a Les usages des cartes, Jean-Marc Besse scriveva:

Depuis une vingtaine d'années, les historiens de la cartographie sont devenus sceptiques vis-à-vis de leur objet. Les influences conjuguées du mouvement de la déconstruction en philosophie, de la sociologie des sciences et des études post-coloniales ont fait de la carte un objet sinon suspect, au moins douteux, et en tout état de cause un objet qui a perdu de l'évidence et de la transparence qui lui étaient accordées

I. L'articolo è il risultato della rielaborazione degli interventi effettuati in occasione del Convegno di San Biagio e del 32nd International Geographical Congress Cologne 2012, Commission History of Geography Pre-Congress and Field Trip «Cartography and Geographical Knowledge in the Public Sphere», Leipzig, Leibniz-Institut für Landerkunde, August 21-26, 2012. 
jusqu'alors. Les historiens de la cartographie se sont accoutumés à travailler sur un objet devenu opaque. A vrai dire, cette nouvelle donne est peut-être une chance pour la cartographie et pour son histoire. Car cette opacité est l'occasion qui permet de restituer aux objets, aux méthodes et aux pratiques cartographiques, leur véritable complexité, c'est-à-dire leur véritable statut de problèmes pour l'historien ${ }^{2}$.

L'autore riprende il discorso qualche tempo dopo, introducendo un numero dei Carnets du paysage tutto dedicato alle "Cartographies» ${ }^{3}$. Nell'uno e nell'altro intervento Besse ci ricorda la rivoluzione epistemologica che negli anni Ottanta aveva investito la carta: considerata fino ad allora in una prospettiva positivista e naturalista, vale a dire come rappresentazione oggettiva della realtà, la carta era stata ridefinita quale operazione retorica, inesatta per natura, strumento di potere. Da Brian Harley, Denis Woodward, Mark Monmonier nel mondo anglosassone, a Christian Jacob in Francia - e, bisogna aggiungere, a Franco Farinelli e Massimo Quaini in Italia - la carta come specchio fedele della realtà era stata messa in discussione ${ }^{4}$. Della carta si era criticata l'ambizione di voler restituire tutto il complesso del sensibile, esaurire per segni l'intera immagine del mondo; si era mostrato come di per sé essa contenga un progetto, esplicito o implicito, sul mondo, un'intenzione di trasformazione o di dominazione.

I due interventi citati (e le due pubblicazioni che li ospitano: ma avrei potuto fare diversi altri esempi), effettuati da un autore che è il direttore della rivista dell'Ecole nationale supérieure du paysage di Versailles sono, evidentemente, assai significativi sia a riguardo della vitalità degli studi cartografici in ambito francese, sia per le connessioni che tali studi hanno con il paesaggio essendone la carta una delle forme privilegiate di rappresentazione. Anzi, Besse si addentra nel processo figurativo distinguendo fra représentation, vale a dire la raffigurazione delle forme paesistiche che possiamo effettivamente osservare nella realtà, e figuration, quando la carta mostra un oggetto che possiamo vedere solo attraverso il suo disegno 5 .

2. J.-M. Besse, «Cartographies et pensée visuelle», p. 19.

3. J.-M. Besse, "Cartographies».

4. Cf. Bibliogafia per le referenze.

5. J.-M. Besse, "Cartographies et pensée visuelle», p. 27. 
L'individuazione dei processi mentali e materiali che hanno prodotto la carta ci riporta alle dinamiche politiche e alle strutture sociali che hanno storicamente interessato lo spazio geografico in essa rappresentato. Con ciò si possono assumere le carte storiche (in particolare alle grandi scale) e la documentazione d'archivio che le contestualizza come fonti di notevole interesse per conoscere, di un dato paesaggio, le trasformazioni e le condizioni che le hanno determinate, e ciò a vantaggio, per esempio, degli strumenti urbanistici quali piani territoriali dei vari livelli e piani paesaggistici.

Nel quadro delle riflessioni qui sommariamente esposte si è sviluppato il mio lavoro sul corpus cartografico di epoca napoleonica che mi accingo a presentare, un insieme documentale di carte, schizzi, acquarelli, plastici da studiare proprio nelle prospettive indicate da Besse:

On n'étudie plus aujourd'hui la carte sans la relier aux pratiques et aux intentions de ses auteurs et de ses destinataires, plus généralement aux contextes pragmatiques de sa production, de sa circulation et de sa consommation [...]. De cet élargissement des questionnements, on peut retirer au moins deux leçons: il n'y a pas de première ni de dernière carte à proprement parler. Toute carte est prise dans un héritage, qu'elle prolonge et transforme. Elle est la pointe provisoirement ultime d'une bibliothèque ou d'une cartothèque. Il n'y a pas de carte sans mémoire (certes, plus ou moins refoulée). Elle est, par ailleurs, une coupe instantanée dans un processus de figuration qui commence dès la première esquisse de terrain, peut être même de la première anticipation, avant tout dessin effectif, avant le rencontre du terrain. En ces sens il n'y a pas lieu, pour le paysagiste en particulier, de séparer de manière absolue la carte de l'ensemble des figurations qui l'accompagnent (croquis, maquettes, diagrammes etc.) ${ }^{6}$.

L'obiettivo principale è stato dunque quello di studiare le "mie» cartografie nel più generale discorso della carta $^{7}$ e, d'altra parte, nel processo di costruzione del paesaggio rappresentato. Nel presente intervento mi soffermerò in particolare sul primo punto per collocare l'insieme

6. J.-M. Besse, «Cartographies», p. 7.

7. Il possessivo richiama il bel saggio di B. Harley, "La carte en tant que biographie». In proposito cfr. L. Rossi, «Alla periferia dell'Impero». 
citato nella lunga di transizione della carta dal linguaggio imitativo al linguaggio astratto.

La discussione su come rappresentare il paesaggio nelle carte può essere letta attraverso questa tensione fra imitazione della natura e astrazione, paesaggio "artistico» e paesaggio "geometrico». A determinare la differenza della grammatica cartografica è il punto di vista adottato: l'analisi prospettiva permette di distinguere le forme dei fenomeni la cui restituzione richiede più le competenze dell'artista che quelle del cartografo. Lanalisi verticale schiaccia il paesaggio, gli toglie ogni profondità, ma consente di mostrare l'organizzazione spaziale dei fenomeni in insiemi omogenei e gerarchizzati. Per tutto il Seicento e oltre la questione è "risolta» in una produzione cartografica che cerca di contenere entrambi i punti di vista attraverso quella che per convenzione è stata definita perspective cavalière. In un bel saggio in cui affronta precocemente (1982) il tema della percezione geografica del rilievo terrestre, Jean-Pierre Nardy parla di un grande seduzione: il progetto di "combiner sur le même document représentation verticale et répresentation perspective, afin d'embrasser d'un seul regard le réel sous tous ses aspects et de lire simultanément un plan et une table d'orientation panoramique» ${ }^{8}$.

Come di ogni seduzione, anche di questa cartografica conviene capire i rischi : come ancora ci ricorda Nardy, la prospettiva alla cavaliera offre, se ben eseguita, una percezione convincente del paesaggio ma è un pessimo strumento di localizzazione. Dunque, se è vero che "smontando" il paesaggio attraverso l'adozione del doppio punto di vista ne risultano figure di più immediata comprensione, è anche vero che tali carte ibride sono lontane dal coerente modello geometrico del mondo che si andava perseguendo.

In questo conflitto fra visione orizzontale e verticale, percezione e misura, è la Francia a dare il maggior contributo in termini teorici, didattici e di prodotti cartografici come testimoniano la vasta trattatistica, gli insegnamenti impartiti nelle scuole militari, le carte realizzate. Anche la Carta dei Cassini, che, completata nel 1793, restituisce alla scala topografica l'intero territorio dello stato $^{9}$, lasciando irrisolto,

8. J-P. Nardy, «Réflexions sur l'évolution historique de la perception géographique du relief terrestre», p. 229.

9. Sulla Carta di Cassini si veda M. Pelletier, Les Cartes des Cassini. 
o piuttosto, risolvendo in modo assai sommario il problema della terza dimensione, è partecipe di questo dibattito. Eliminato il punto di vista orizzontale, nelle tavole della prima Carta di Francia le catene delle montagne sono rese con un tratteggio che John Wilford Noble ha definito "bruchi pelosi ${ }^{10}$. In termini meno scherzosi Dainville aveva ben chiarito i limiti della carta cassiniana. Basta dare un'occhiata ai fogli che raffigurano le zone più montuose, aveva scritto, per notare lo scarto fra il rigore dell'impianto astronomico-geometrico e la nullità, perfino «stupefacente", della rappresentazione dei rilievi. Del resto, come si evince da una sua lettera del 1784, Cassini non fa mistero di aver deliberatamente deciso di fare una carta geometrica e non topografica: la topografia, che offre la descrizione dettagliata e scrupolosa della conformazione del terreno e dei contorni esatti delle vallate, delle montagne, delle coste, è una parte della geografia talmente estesa, minuziosa, lunga e costosa nell'esecuzione, che non poteva ancora essere affrontata in una carta generale. Come riporta Dainville, lo stesso Cassini aveva scritto:

On n’a jamais prétendu en faire qu'un accessoire à la Carte générale de la France. Pour la rendre plus agréable, on y a joint, pour ainsi dire, une esquisse, une ébauche de topographie ${ }^{11}$.

Sui possibili modi di rappresentare il rilevo eliminando la prospettiva orizzontale si discuterà a lungo fra il XVIII e il XIX secolo: alla fine resteranno sostanzialmente sul tappeto le linee di massima pendenza (hachures) e le curve di livello (courbes de niveau). L'invenzione delle isoipse, maturata negli ambienti delle carte marine nella seconda metà del Settecento, non comporta la loro rapida adozione se non per siti molto circoscritti tanto che per la generalizzazione del metodo bisognerà attendere la seconda metà dell'Ottocento.

Principalmente intorno alle questioni ricordate - messa in discussione della prospettiva orizzontale e dibattito sulla rappresentazione del rilievo - va letta la vicenda delle curve di livello che abbraccia appunto buona parte del Settecento e della prima metà dell'Ottocento : quali furono le prime carte realizzate con tale metodo, in quale circostanza, sulla base di quali argomentazioni, ecc. Date le numerose implicazioni

Io. J.W. Noble, Cartografi, p. 114.

II. F. de Dainville, «De la profondeur à l'altitude», p. 195 sq. 
del problema, mi limiterò a fornire le principali indicazioni utili a inquadrare il caso di studio qui presentato.

Siamo alla fine degli anni Venti dell'Ottocento, e precisamente nel 1826-1827 e nel 1828 quando hanno luogo le sedute della commissione, creata per discutere del rinnovamento delle tecniche topografiche e della necessità di uniformare il linguaggio delle carte, presieduta dal generale del Genio Dode de la Brunerie. Veniva cioè ripreso ufficialmente dalle istituzioni il discorso avviato nel 1802 quando la prima (e più famosa) commissione di esperti dei vari servizi militari e civili dello stato era stata riunita allo stesso scopo ${ }^{12}$. Allora la discussione era avvenuta nell'esaltante clima napoleonico, con tutto quello che esso ha significato anche in fatto di produzione di documenti cartografici sia relativi ai campi di battaglia sia all'attività del Genio. In piena Restaurazione la commissione citata prosegue il discorso sulla topografia sotto la spinta della realizzazione di una nuova Carta di Francia capace di replicare, con tutti gli adeguamenti necessari, la grande impresa dei Cassini.

Come quasi un trentennio prima, il Mémorial del Dépôt de la guerre è il portavoce dei risultati dei lavori. Nel volume relativo agli anni 1827 e 1828 troviamo riportata la Note del colonnello del corpo degli Ingegneri geografi Bonne:

Nous ne devrions pas perdre de vue qu'une carte topographique doit être une espèce de tableau. Le sujet est par lui-même très aride; ne lui enlevons pas le peu d'agrément dont il est susceptible ${ }^{13}$.

Infatti, le questioni al centro della discussione non sono molto cambiate dal 1802 quando la commissione aveva concluso le sedute liquidando del tutto la prospettiva cavaliera e lasciando aperti diversi problemi, sopratutto relativi al figuré. La necessità di non eliminare dalla carta quel «peu d'agrément" capace di fornire la percezione immediata del paesaggio aveva determinato la diversità delle posizioni prese nel tempo da personaggi come lo stesso Bonne, il suo collega Puissant, o il generale del Genio Haxo, e aveva portato a riconsiderare l'uso delle ombre portate e della luce (obliqua o verticale) e a continuare preferire, per rappresentare

I2. Mémorial topographique et militaire rédigé au Dépôt Général de la Guerre, N. 5, an XI.

13. Mémorial du Dépôt Général de la Guerre, Tome V, p. 462. 
l'altitudine, le hachures ${ }^{14}$. Che le curve di livello, derivate dal preciso rilevamento delle montagne e dalla loro restituzione in pianta corrispondessero all'esigenza di esattezza e omogeneità della carta era chiaro a tutti; i dubbi riguardavano se esse potessero essere applicate senza enorme impiego di tempo e denaro a territori vasti (tenendo conto del fatto che le prime sperimentazioni, per esempio quelle relative alla fortificazione di Rocca d'Anfo a Brescia progettata negli anni 1801-1802 da Haxo e Liédot, erano circoscritte alla scala di sito ${ }^{15}$ ); quanto eventualmente distanziare le linee che congiungevano i punti di una stessa quota; quando utilizzarle sole e quando associarle all'uso del tratteggio o del colore per rendere la rappresentazione delle forme terrestri più facilmente percepibile, ecc.

La nuova commissione ha dunque il compito di «examiner parmi les différents systèmes suivis ou proposés pour représenter géométriquement et physiquement le relief du terrain, quel est celui qui doit être adopté généralement", come si evince dalle istruzioni trasmesse dal ministro della guerra Clermont Tonnerre al presidente Dode de la Brunerie in data 25 febbraio $1826^{16}$. Memore delle motivazioni che nel 1802 avevano fatto recedere dall'adozione del metodo delle isoipse, l'indicazione è che la commissione «devra rechercher en même temps s'il est applicable à toutes les échelles, et particulièrement à celle de 1 pour 40.000 [scala adottata per le minute della Carta di Francia] et du 1 pour 80.000 [scala per la stampa] ${ }^{17}$. Si raccomanda che il nuovo sistema

I4. Il Dépôt de la guerre, l'Ecole Polytechnique e l'Ecole de Saint Cyr si erano adeguati alle indicazioni della Commissione, diversamente dalla scuola del Genio di Metz, convinta della maggiore razionalità della luce verticale; l'uso di questa si afferma sempre di più anche la Scuola Politecnica che lo adotta ma, essendo ancora le indicazioni del 1802 in vigore, la confusione regnava sovrana. Inviato in ispezione a Metz, Haxo scrive due memorie in cui si dichiara contrario alla luce obliqua ma anche contro le curve orizzontali per sostenere una laboriosa adozione delle hachures. Molti ingegneri geografi come, del resto, gli ufficiali di Stato maggiore, si erano rifiutati di utilizzare il metodo della luce obliqua. Puissant, membro della Commissione reale per la nuova carta di Francia, aveva scritto un'apposita memoria in difesa della luce obliqua (Observations sur les diverses manières d'exprimer le relief..., 1818); su posizioni opposte, Bonne aveva risposto sostenendo l'uso della luce verticale integrata con la differente intensità delle hachures secondo il grado di inclinazione delle pendenze (Supplément aux considérations..., 1818). Il dibattito è riportato in Mémorial du Dépôt Général de la Guerre, Tome IV, p. 347 sq.

I5. L. Rossi, "Verso le isoipse», in corso di stampa.

16. Mémorial du Dépôt Général de la Guerre, Tome IV, p. 351.

17. Ibid. 
non superi i costi del precedente; che la rappresentazione delle montagne ne risulti di lettura semplice e immediata e che non se ne sacrifichi alcun dettaglio e neppure la disposizione e la chiarezza dei toponimi. Il ministro conclude le istruzioni dicendo:

Je vous ferai très-incessamment l'envoi des divers dessins-modèles de gravure dans deux systèmes, et qui pourront être mis sous les yeux de la commission. Quelques mémoires sont à l'appui, et le tout a été exécuté au Dépôt de la Guerre; j’y joindrai des exemples des différents systèmes employés par les étrangers ${ }^{18}$.

Per poter meglio discuterne, «sotto gli occhi» dei componenti della commissione vengono dunque messi dei modelli di carte che troviamo regolarmente stampati nello stesso Mémorial. Sul supporto ripetuto di una carta vengono rappresentate le principali modalità per raffigurare il rilievo del terreno. Il tema sono ancora le courbes e le hachures. Soffermarsi sull'identità del supporto non è lo scopo di queste figure di cui non vengono infatti indicati né la data, né l'autore. L'annotazione «Dessiné par Desmadryl ainé» non si riferisce all'autore della carta-base ma soltanto al redattore della "prova».

Ma da dove derivano queste figure? "Tout a été exécuté au Dépôt de la Guerre" aveva detto il ministro ${ }^{19}$. In realtà, se le "prove» erano state elaborate al Dépôt de la Guerre, la base cartografica è una carta molto speciale, frutto dell'attività del Dépôt des fortifications di quasi trent'anni prima, e non sembra un caso che sia stata proprio questa carta a fare da supporto all'esercizio. Il documento, di cui non abbiamo ancora ricostruito attraverso quali precisi passaggi sia trasmigrato dai servizi del Genio a quello della Guerre, di sicuro ci rimanda all'epoca napoleonica e al capitano del Genio Pierre-Antoine Clerc (Nantua 1770Metz 1843). Dopo aver prestato servizio sui campi di battaglia, si era dedicato alla topografia, nel 1801 era stato chiamato a Parigi dal ministro Carnot, aveva partecipato alla Commissione del 1802 e solo negli anni successivi, sia sul terreno (negli anni fra il 1805 e il 1808 si misura con le courbes sul terreno parigino), sia come professore di topografia all'Ecole Polytechnique, aveva iniziato a praticare il metodo delle isoipse

I8. Ibid., p. 352.

19. Ibid. 
diventandone il più convinto sostenitore ${ }^{20}$. La sua carte nivelée del promontorio di Porto Venere (la Spezia) risulta essere, allo stato attuale della ricerca, la prima carta a curve di livello della storia della cartografia raffigurante non un sito circoscritto ma un territorio piuttosto esteso. Eseguita nel 1812 come riduzione al 5000 dei rilevamenti al 1000, alla soglia degli anni Trenta dell'Ottocento essa doveva aver acquisito lo statuto di carta simbolo su cui verificare una discussione ancora irrisolta.

Nel 1808 Napoleone ordina che venga realizzato il plastico del territorio del dipartimento del Levante ligure su cui vuole far sorgere il più grande arsenale del Mediterraneo e una nouvelle ville; l'incarico viene affidato Clerc che viene inviato sul terreno insieme ad allievi topografi e a un paio di della Galerie des plans-reliefs. È questo il primo nucleo della brigade topographique del Dépôt des Fortifications creata nel 1809 e ufficializzata nel 1813, della quale Clerc resterà a capo fino al 1827 ma che avrà lunga vita anche dopo di lui.

Sul tema delle isoipse è intervenuto, negli ormai lontani anni Cinquanta del Novecento, Dainville in modo significativo dal punto di vista interpretativo e perfino affascinante dal punto di vista letterario per l'immagine che lo studioso gesuita chiama in causa: quella di Venere che sorge dal mare così come la nascita delle isoipse va fatta risalire alla pratica delle sonde. "Il n'est point banal que les courbes de niveau de nos cartes sortent de l'onde, telle Aphrodite, pour monter jusqu'aux neiges éternelles ${ }^{21}$. Dainville aveva declinato la sua analisi essenzialmente sulla genesi delle curve senza mancare di segnalare le prime e più significative esperienze di pratica di terreno, esperienze da lui appena citate ma che suggerivano di continuare con specifiche ricerche a valle del suo discorso. Quanto al mio interesse per l'argomento, esso non ha preso le mosse dal saggio di Dainville, a cui sono arrivata solo dopo la scoperta della ricchissima documentazione riguardante una delle esperienze che il père aveva indicato come fondamentale nel processo di affermazione delle courbes nelle carte terrestri: il lavoro effettuato da Clerc nel territorio (casualmente denominato proprio "Porto di Venere») oggetto dei miei studi di geografa ${ }^{22}$.

20. L. Rossi, "Pierre-Antoine Clerc, un topografo di rilevo fra arte e misura», in corso di stampa.

2I. F. de Dainville, "De la profondeur à l'altitude», p. 209.

22. L. Rossi, «Paesaggi e memoria». 
La Spezia rappresenta dunque il primo laboratorio della brigata di Clerc che nel territorio del Golfo non produce solo carte a curve di livello ma anche una vasta documentazione vedutistica. Dopo l'esperienza spezzina la brigata verrà inviata in altri territori-chiave nella politica napoleonica: Cherbourg, Mont Cenis, Hyères (1812-13), Colonia e Coblenza $(1814)^{23}$; qualche traccia archivistica consente di ipotizzare che anche in queste missioni la brigata abbia operato con il metodo messo a punto in Liguria anche se fino a noi sono arrivati (in un certo senso fortunosamente ${ }^{24}$ ) soltanto schizzi e acquarelli di paesaggio della prima spedizione. Il corpus che la riguarda è infatti composto nel suo insieme da tre distinte tipologie di rappresentazioni $^{25}$ : piante e carte ${ }^{26}$, disegni in prospettiva orizzontale ${ }^{27}$, modelli tridimensionali ${ }^{28}$. L'inquadramento di questi materiali nella specifica spedizione che li ha prodotti, i collegamenti interni ed esterni che li

23. Per un quadro sommario delle missioni della brigata e, invece, un quadro sufficientemente dettagliato degli archivi che ne conservano la documentazione cartografica cfr. L. Rossi, "La brigade topographique et ses archives".

24. L. Rossi, "La vera storia dei Cartografi Imperiali", e in particolare il paragrafo «Il ritorno alla Spezia di disegni troppo belli», p. 44-49.

25. Del magnifico corpus vedutistico e cartografico si possono vedere numerose immagini e tutte le schede analitiche in L. Rossi, Napoleone e il Golfo della Spezia, p. 54-237.

26. Si tratta di piante di manufatti a grandissima scala, delle tavolette di campagna acquarellate in scala 1:1000 e della carta definitiva del promontorio di Porto Venere e isole rilevata fra il 1809 e il 1811 pure in scala 1:1000 (quadro di unione e 18 fogli). Qui ogni elemento topografico è restituito con estremo dettaglio: edifici, muri a secco, scalini che collegano un terrazzamento all'altro sono rappresentati uno ad uno (in rosso). «Chiude» il gruppo delle planimetrie la carte nivelée, di cui si pubblica un particolare (fig. 1), riduzione effettuata nel 1812 alla Galerie della precedente, ma non comprensiva delle isole. In questa figura domina la rappresentazione del rilievo, restituito con esattezza dalle curve di livello. Nei tratti più scoscesi, là dove le coste si frammentano in anfratti e piccoli scogli, il disegno imitativo reso in minuti tratti a penna, acquarellato in grigio, trasmettere con intensità la percezione del rilievo. Parigi, Musée des plansreliefs, Archives.

27. Il "catalogo" è ricco e variegato: si va dagli alzati dei manufatti, agli schizzi di paesaggio, alle belle vedute (fig. 2) (acquarellate e non). Parigi, Musée des plans-reliefs, Archives e La Spezia, Biblioteca Civica «U. Mazzini».

28. Furono realizzati tre plastici: quello dell'intero promontorio e delle isole non rivestito della vegetazione, in scala 1:1000 (7,53 x 4,04 m) (fig. 3); la versione "vestita" della vegetazione di una parte del precedente, stessa scala $(2,58 \times 1,58 \mathrm{~m})$; stessa scala anche per il piccolo modello dell'Isola del Tino (0,72 x 0,45 m). Parigi, Musée des plansreliefs, Archives. 
Fig. 1 - Brigata topografica di P.-A. Clerc, Carte Nivelée par courbes horizontales. Presqu'île de Castellana et Porto-Venere dans le Golfe de la Spezzia levée pour servir à l'exécution de la Carte-Relief ordonnée par Sa Majesté, 1812. Disegno a china nera e rossa, acquarellato policromo, scala $1: 5000,91,5 \times 94 \mathrm{~cm}$. Particolare. 
Fig. 2 - Brigata topografica di P.-A. Clerc, Veduta di Porto Venere dall'Isola Palmaria, 1809-1811. Disegno a china nera acquarellato policromo, 30 x $46 \mathrm{~cm}$.

riguardano, e più in generale la loro collocazione nelle tendenze della cartografia dell'epoca costituiscono l'oggetto del mio studio ancora in corso.

Le considerazioni che si possono anticipare riguardano appunto le connessioni fra le tre tipologie indicate. Carte a curve di livello, vedute, plastici, costituiscono categorie diverse di rappresentazioni all'interno di un'operazione che, essendo solo indirettamente finalizzata alla costruzione dell'arsenale spezzino in quanto direttamente finalizzata alla costruzione della maquette del sito in cui il manufatto doveva sorgere, tutte le intreccia e, d'altra parte, spiega l'abbondanza e il dettaglio della documentazione di carattere artistico. Ai precisi rilievi topografici per le carte si associa il vedutismo utile a memorizzare il paesaggio per il plastico la cui lavorazione sarebbe stata ultimata a Parigi. E non è da sottovalutare il fatto che, prima di aver interpellato Clerc, le istituzioni si fossero rivolte, per la realizzazione del modello tridimensionale voluto da Napoleone, al famoso pittore di vedute di battaglie Giuseppe Pietro Bagetti e a suo cognato Pasquier: "scelta significativa in quanto ambedue erano ritenuti avere più esperienza artistica che 
Fig. 3 - Brigata topografica di P.-A. Clerc, Spezia sur le Golfe de Gênes (Italie), 1811. Plastico policromo, scala 1:1000, 7,53 × 4,04 m. Particolare del Promontorio di Porto Venere.

topografica» ${ }^{29}$. A proposito di «topografi artisti», Massimo Quaini ha scritto:

Serve innanzitutto sapere che cosa si intendesse per artista a cavallo fra Sette e Ottocento e se e quanto la contestuale concezione del bello (quando ancora non si parlava di estetica) potesse contemplare anche il lavoro artigianale del cartografo. Serve poi verificare entro quali limiti i cartografi partecipassero alla multiforme cultura paesaggistica di un'epoca che scopre i paesaggi alpini : cosa non secondaria per una cartografia che si pone il problema di una nuova rappresentazione del rilievo montuoso ${ }^{30}$.

29. M. Quaini, "Quando il cartografo era un artista», p. 26.

30. Ibid., p. 19. 
Il saggio citato è prezioso per interpretare l'attività della brigade. Certo è che l'aver affidato a Clerc, ormai conosciuto per le sue convinzioni in merito alle curve di livello, il compito di realizzare il plastico è l'atto che coniuga un sapere molto avanzato dal punto di vista tecnico e la tradizione topografico-pittorica, con la conseguenza di una rilevante produzione cartografica ricca di connessioni fra moduli geometrici e moduli artistici.

Del resto il plan en relief è, di per sé, oltre che una "carta", una scultura modellata, una carta speciale che consente senza contraddizioni - senza la "conversione mentale» cui la carta alla cavaliera obbligava l'osservatore - di praticare direttamente l'osservazione orizzontale e verticale, "de situer un objectif dans l'espace et identifier un élément topographique dans le panorama» ${ }^{31}$.

Lo stesso luogo che sovrintendeva alla costruzione e conservazione dei plans-reliefs rinvia, anche nel nome, a uno spazio di collezioni d'arte: la Galerie. Creata all'epoca di Luigi XIV al Louvre per collocarvi i plastici delle fortificazioni vaubaniane ${ }^{32}$, oggetti di confine fra saperi topografici e arti plastiche e, d'altra parte, fra necessità strategiche e intenti comunicativi, con Napoleone essa conosce una nuova vitalità ${ }^{33}$. Dalla Galerie, all'epoca trasferita agli Invalidi (dove ancora oggi si trova), provengono alcuni degli uomini della brigade topographique affidata nel 1809 a Clerc per la specifica spedizione in Liguria. La composizione della brigata, fatta di un capitano che era anche professore di topografia, di soldati del Genio abituati a misurare il terreno e, appunto, di artisti in forze alla stessa galleria, è il riassunto dei saperi cartografici a cavallo fra Sette e Ottocento, il trasferimento sul campo di un dibattito in corso.

Nella lunga tradizione dei plans en relief, quello spezzino è dunque il primo ad essere realizzato sulla base delle curve di livello. Rilevamenti planimetrico-altimetrici da una parte e riprese vedutistiche dall'altra, tutto doveva servire a fare, se pur in scala, lo spettacolare doppio (di cui forniamo qui solo un particolare) di un paesaggio di per sé spettacolare,

3I. J.-P. Nardy, «Réflexions sur l'évolution historique de la perception géographique du relief terrestre», p. 229.

32. I. Warmoes, Le musée des Plans-Reliefs.

33. Sul significato dei plastici e sul ruolo della Galerie è in corso l'ottimo studio, incentrato sul Plan en relief du Siège de Rome (1849-1852), di Valentina De Santi (dottorato Geografia storica, Università degli Studi di Genova e Ecole des Hautes Etudes en Sciences Sociales, Paris). 
sia nelle falesie a strapiombo sul mare, sia nei versanti elevati ma addomesticati dai terrazzamenti. Ma l'esercizio effettuato per riprodurre nelle decine e decine di schizzi e nelle vedute acquarellate le forme del terreno, il ricamo delle rocce, i colori delle acque, della terra, della vegetazione, non poteva mancare di riversarsi almeno in parte sia nelle tavolette di campagna nelle quali le curve di livello solcano un paesaggio minuziosamente analizzato attraverso il colore, sia nelle planimetrie finali.

Se «l'ossessione» delle istituzioni e degli addetti ai lavori della prima metà dell'Ottocento era, come si evince dalla lettura dei vari volumi del Mémorial e della trattatistica dell'epoca, la realizzazione di carte che, accantonata l'ambiguità spaesante della veduta alla cavaliera, restituissero il paesaggio in pianta senza perdere la percezione immediata delle forme terrestri, mi pare di poter dire che nel primo decennio del secolo il capitano Clerc, se pur ancora relativamente a una scala topografica non piccola, era riuscito nello scopo.

Luisa Rossi

Università di Parma 


\section{BIBLIOGRAFIA}

Besse, Jean-Marc, "Cartographies et pensée visuelle. Réflexion sur la schématisation graphique", in Les usages des cartes (XVII ${ }^{e}$ $X I X^{e}$ siècle). Pour une approche pragmatique des productions cartographiques, dir. par Isabelle Laboulais, Strasbourg, Presses Universitaires de Strasbourg, 2008, p. 20-32.

—, "Cartographies. Editorial», Les Carnets du Paysage, 20 (automnehiver 2010-2011), p. 5-9.

Dainville, François de, «De la profondeur à l'altitude. Des origines marines de l'expression cartographique du relief terrestre par cotes et courbes de niveaux", in Le Navire et l'économie Maritime du Moyen Age au XVIII e siècle principalement en Méditerranée. Travaux du Deuxième Colloque international d'histoire maritime, dir. par Michel Mollat, Paris, Bibliothèque générale de l'Ecole pratique des Hautes Etudes, 1958 (1959), p. 195-222. (Pubblicato una seconda volta in François de Dainville, La cartographie reflet de l'histoire, Genève/Paris, Editions Slatkine, 1986, p. 441-457).

Farinelli, Franco, I segni del mondo. Immagine cartografica e discorso geografico in età moderna, Aqui Terme, Academia Universa Press, 2009 (prima edizione: Firenze, La nuova Italia, 1993).

Gould, Peter, Bailly, Antoine (éd.), Le pouvoir des cartes. Bryan Harley et la cartographie, Paris, Anthropos, 1995.

Harley, Brian, "La carte en tant que biographie", in Le pouvoir des cartes. Bryan Harley et la cartographie, édité par Peter Gould et Antoine Bailly, Paris, Anthropos, 1995, p. 11-18.

HouellebecQ, Michel, La carte et le territoire, Paris, Flammarion, 2010. Јасов, Christian, "Sables, neiges, eaux", in Cartes et figures de la terre, Paris, Centre Georges Pompidou, 1980, p. 220-221.

—, L'empire des cartes. Approche théorique à la cartographie à travers l'histoire, Paris, Albin Michel, 1992.

Mémorial du Dépôt Général de la Guerre, Tome IV, Année 1826, Paris, Piquet, 1828, p. 345-391 e carte. 
Mémorial du Dépôt Général de la Guerre, Tome V, Années 1827 et 1828, Paris, Piquet, 1829, p. 458-485.

Mémorial topographique et militaire rédigé au Dépôt Général de la Guerre, N. 5, "Topographie», Paris, Imprimerie de la République, Fructidor an XI [septembre 1803].

Monmonier, Mark, Comment faire mentir les cartes. Du mauvage usage de la géographie, Paris, Flammarion, 1993.

Nardy, Jean-Pierre, "Réflexions sur l'évolution historique de la perception géographique du relief terrestre", L'Espace Géographique, 3 (1982), p. 224-232.

Noble, John Wilford, Cartografi, Milano, Edizioni Sylvestre Bonnard, 2005.

Pelletier, Monique, Les Cartes des Cassini. La science au service de l'Etat et des régions, Paris, Editions du C.T.H.S, 2002.

Quaini, Massimo (a cura di), Il mito di Atlante. Storia della cartografia occidentale in Età Moderna, Genova/Trento, Il Portolano/Centro Studi Martino Martini, 2006.

—, "Quando il cartografo era un artista», in Napoleone e il Golfo della Spezia. Topografi francesi in Liguria tra il 1809 e il 1811, a cura di Luisa Rossi, Cinisello Balsamo, Silvana Editoriale, 2008, p. 19-30.

Rossi, Luisa, "Alla periferia dell'Impero : operazioni topografiche francesi nel Golfo della Spezia", in Cartografia e progettazione territoriale. Dalle carte coloniali al piano, a cura di Emanuela Casti, Torino/Novara, UTET Università/De Agostini, 2007, p. 115-132.

- (a cura di), Napoleone e il Golfo della Spezia. Topografi francesi in Liguria tra il 1809 e il 1811, Cinisello Balsamo, Silvana Editoriale, 2008.

—, "La vera storia dei Cartografi Imperiali», in Napoleone e il Golfo della Spezia. Topografi francesi in Liguria tra il 1809 e il 1811, a cura di Luisa Rossi, Cinisello Balsamo, Silvana Editoriale, 2008, p. 31-49.

—, «Paesaggi e memoria. Il nome, il disegno del territorio, l'utopia della città ", in Il senso del Golfo. Dalla foce della Magra alle Cinque Terre, a cura di Rossana Piccioli, Alessandro Scansani, Reggio Emilia, Diabasis, 2008, p. 45-76.

—, "La brigade topographique et ses archives", in Le Dépôt des fortifications et ses archives 1660-1940, dirigé par Nicole Salat, Emmanuel Pénicaut, Paris, Ministère de la Defense/Archives et Culture, 2011, p. 97-113. 
—, "Verso le isoipse», in Di monti e di acque. Paesaggi, cartografie e modi del discorso geostorico, a cura di Elena Dai Prà, Milano, Angeli Editore, in corso di stampa.

—, "Pierre-Antoine Clerc, un topografo di rilevo fra arte e misura", in Atti del Seminario di studi Storie di cartografi, storia della cartografia. La biografia nella ricerca geografica, Torino 8-9 giugno 2006, in corso di stampa.

Warmoes, Isabelle, Le musée des Plans-Reliefs. Maquettes historiques de villes fortifiées, Paris, Editions du Patrimoine, 1997.

\section{Referenze iconografiche}

Fig. 1:

Parigi, Musée des Plans en relief, Archives, INV. 772 - Art. IV, n. E, carton 4 : p.g.c.

Foto: Bruno Arrigoni 2008.

Fig. 2:

Comune di Porto Venere, p. g. c.

Foto : Enrico Amici, 2008.

Fig. 3:

Parigi, Musée des Plans en relief, Archives (non esposto) : p. g. c.

Foto: Bruno Arrigoni 2008. 